\title{
Role of PhaC Type I and Type II Enzymes during PHA Biosynthesis
}

\author{
Valeria Mezzolla ${ }^{1}$ (D), Oscar Fernando D'Urso ${ }^{1}$ and Palmiro Poltronieri ${ }^{2, *}$ (i) \\ 1 Department of Biological and Environmental Science and Technologies, University of Salento, Ecotekne, \\ 73100 Lecce, Italy; valeria.mezzolla@gmail.com (V.M.); bioesplora@gmail.com (O.F.D.) \\ 2 CNR, Agrofood Department, Institute of Sciences of Food Productions (ISPA-CNR), 73100 Lecce, Italy \\ * Correspondence: palmiro.poltronieri@ispa.cnr.it; Tel.: +39-0832-422609
}

Received: 26 July 2018; Accepted: 9 August 2018; Published: 13 August 2018

\begin{abstract}
PHA synthases (PhaC) are grouped into four classes based on the kinetics and mechanisms of reaction. The grouping of PhaC enzymes into four classes is dependent on substrate specificity, according to the preference in forming short-chain-length (scl) or medium-chain-length (mcl) polymers: Class I, Class III and Class IV produce scl-PHAs depending on propionate, butyrate, valerate and hexanoate precursors, while Class II PhaC synthesize mcl-PHAs based on the alkane (C6 to C14) precursors. PHA synthases of Class I, in particular PhaC $\mathrm{C}_{\mathrm{Cs}}$ from Chromobacterium USM2 and $\mathrm{PhaC}_{\mathrm{Cn}} / \mathrm{RePhaC} 1$ from Cupriavidus necator/Ralstonia eutropha, have been analysed and the crystal structures of the C-domains have been determined. $\mathrm{PhaC}_{\mathrm{Cn}} / \mathrm{RePhaC} 1$ was also studied by X-ray absorption fine-structure (XAFS) analysis. Models have been proposed for dimerization, catalysis mechanism, substrate recognition and affinity, product formation, and product egress route. The assays based on amino acid substitution by mutagenesis have been useful to validate the hypothesis on the role of amino acids in catalysis and in accommodation of bulky substrates, and for the synthesis of PHB copolymers and medium-chain-length PHA polymers with optimized chemical properties.
\end{abstract}

Keywords: PhaC synthase; classification; dimerization; substrate binding; exit cavity; C3-C14 alkanes; polymer composition

\section{Introduction}

Polyhydroxyalkanoates (PHAs) are biodegradable polyesters produced in several Gram-negative and Gram-positive bacteria, and in archaea and cyanobacteria [1-8]. PHAs are polymers containing various 3-hydroxyalkanaotes, such as 3-hydroxypropionate (3HP), 3-hydroxybutyrate (3HB), 3-hydroxyvalerate $(3 \mathrm{HV}), 3$-hydroxyhexanoate $(3 \mathrm{HHx}), 3$-hydroxyheptanoate $(3 \mathrm{HHp}), 3$-hydroxyoctanoate $(3 \mathrm{HO})$, 3-hydroxynonanoate (3HN), 3-hydroxydecanoate (3HD), 3-hydroxydodecanoate (3HDD), in addition to 4-hydroxybutyrate $(4 \mathrm{HB})$ or 5 -hydroxyvalerate $(5 \mathrm{HV})$, and are produced through the availability of the corresponding CoA thioester substrates [9-14].

There also exist PHAs with unsaturated monomers (3-hydroxyalkenoates), produced by Pseudomonas sp. possessing Class II PHA polymerizing enzymes. PhaC synthases are grouped into four classes based on substrate specificity, and the preference in forming short-chain-length (scl) or medium-chain-length (mcl) polymers: Class I, Class III and Class IV produce principally scl-PHAs, while Class II PhaC synthesize mcl-PHAs [7,15].

The PHA biosynthesis genes include: PhaPs, genes coding for phasins, the granule-assembling proteins; PhaM, encoding the activator and accelerator of the catalytic activity of PHA synthase, encoded by PhaC; phaA, encoding the acetoacetyl-CoA 3-ketothiolase; phbB, coding for the acetoacetyl-CoA reductase; 
phaG, coding for 3-hydroxyacyl-carrier protein-CoA transferase; phaJ, encoding the enoyl-CoA hydratase; and PhaZ, encoding the intracellular PHA-depolymerizing enzymes.

PhaC proteins possess the so-called lipase box, G-X-S-X-G, and a secondary structure containing the $\alpha / \beta$ hydrolase fold, a characteristic succession of alpha helices and beta strands, typical of lipases. In PhaC enzymes, the lipase box has the conserved sequence G-G/S-X-C-X-G/A-G, renamed the PhaC box consensus sequence. The Cys in the lipase box-like sequence is the catalytic amino acid, forming the covalently bonded intermediate, Cys-S-H3B $[7,16]$.

PHA synthase activity is based on the catalytic triad C-H-D, cysteine, histidine, aspartate, also responsible for catalysis in lipases (S-H-D). In the catalytic triad, the negatively charged Asp $_{447}$ in $\mathrm{PhaC}_{\mathrm{Cs}}$ from Chromobacterium spp. $[17,18]$ and $\mathrm{Asp}_{480}$ in $\mathrm{PhaC}_{\mathrm{Cn}}$ from Cupriavidus necator $[19,20]$ assist to enhance the basicity of $\mathrm{His}_{477}$ and $\mathrm{His}_{508}$, respectively, by formation of a direct hydrogen bond. Previous reports suggest that the Asp residue of the triad acts as a general base catalyst to accelerate deprotonation of the 3-hydroxyl group of HB in the step involving elongation of the PHA product.

PHA synthases are grouped into four classes based on the kinetics and mechanism of reaction. The grouping of PhaC enzymes into each class is dependent on the structure of the PhaC, alone or in association with other subunits, and the substrate specificity: Class I, Class III and Class IV produce scl-polymers depending on 3-hydroxypropionate (3HP), 3-hydroxy- and 4-hydroxybutyrate $(3 \mathrm{HB}, 4 \mathrm{HB}), 3$-hydroxyvalerate $(3 \mathrm{HV})$ and 3-hydroxyhexanoate $(3 \mathrm{HH})$ precursors $(\mathrm{C} 3$ to $\mathrm{C} 6$ carbons), while Class II PhaC enzymes synthesize mcl-polymers depending on 3-hydroxyhexanoate $(3 \mathrm{HH}), 3$-hydroxyheptanoate $(3 \mathrm{HHp}), 3$-hydroxyoctanoate (3HO), 3-hydroxydecanoate (3HD), 3-hydroxyundecanoate (3HUD), 3-hydroxydodecanoate (3HDD) (C6 to C12), and availability of the corresponding CoA thioester substrates, originating from three different metabolic pathways $[9,10,20]$.

While some bacterial species produce mainly $\mathrm{PHB}$ homopolymers consisting only of $3 \mathrm{HB}$ monomers, other species can synthesize various PHAs, depending on availability of intermediate precursors $[1,3,9]$. The structure and properties of the polymers are affected by the monomers that are incorporated. PHAs can be molded into films and hollow bodies. Polyhydroxybutyrate (PHB) is brittle, fragile and stiff, with low elongation ability, and a break point below $15 \%$. The incorporation of 3-hydroxyvalerate or other comonomers can decrease the brittleness of PHB. The thermal, rheological and barrier properties of PHAs show good application potential in thermoplastic materials. The synthesis of copolymers is a frequent strategy to improve the properties of PHAs, to improve the plastics flexibility and lower the glass transition temperature $\left(T_{\mathrm{g}}\right)$ and the melting temperature $\left(T_{m}\right)$ [21]. P3HB-4HB polymers and conventional thermoplastics used for packaging show high tensile strength and higher elongation at break. mcl-PHAs containing both $3 \mathrm{HB}$ and $3 \mathrm{HV}$ monomers are elastic, have low melting point, a relatively low degree of crystallinity, and various tensile strengths. PHB copolymer containing 3-hydroxyvalerate unit $\mathrm{P}(3 \mathrm{HB}-\mathrm{co}-3 \mathrm{HV})$ has been developed with improved mechanical properties. To this aim, either optimization of substrate availability (feedstock, successive addition of precursors) or efficiency of enzymes, through genetic engineering and selection of PHA synthases, have been applied.

\subsection{Class I and Class II PHA Synthases}

Classes I and II PHA synthases are formed by a single protein (PhaC), of about $60 \mathrm{kDa}$.

In Class I, the active PhaC enzyme is a dimer, with catalytic (CAT) domains facing each other, and with $\mathrm{N}$-domains making direct contacts, sustaining protein interaction and dimerization. It was hypothesized that a partially folded catalytic domain is partially occupied by the lid-and-cat domain secondary structure, which changes its conformation in the presence of activation factors to open the catalytic domain and to allocate the 3HB-CoA to its binding site [17].

PhaC sequences differ in their length. In Cupriavidus necator (formerly Ralstonia eutropha) $\mathrm{PhaC}_{\mathrm{Cn}} / \mathrm{RePhaC}$, a Class I enzyme, the sequence is composed of a 191 amino acids proteolytically cleaved at the N-terminal domain after arginine, and a C-domain, containing the catalytic site, composed of 398 amino acids, for a total of 589 residues (Figure 1). In C. necator, $\mathrm{PhaC}_{\mathrm{Cn}}$ contains $\mathrm{Cys}_{319}$, 
Asp $_{480}$ and $\mathrm{His}_{508}$, located between the beta- 6 and alpha- 3 turns, the beta-10 and alpha- 7 turns, and at the end of the beta-11 strand, respectively. In between $\mathrm{Cys}_{319}$ and $\mathrm{Asp}_{480}$ is located the D-loop and the helix-turn-helix motif (HTH), formed by $\alpha 4, \alpha 5, \alpha 6$ and $\beta 7-\beta 8$ stretches [18,19]. PhaC $\mathrm{Cn}_{1} / \mathrm{RePhaC} 1$ was also studied by X-ray absorption fine-structure (XAFS) analysis, which confirmed the previous findings on the protein assembled as a dimer [22].

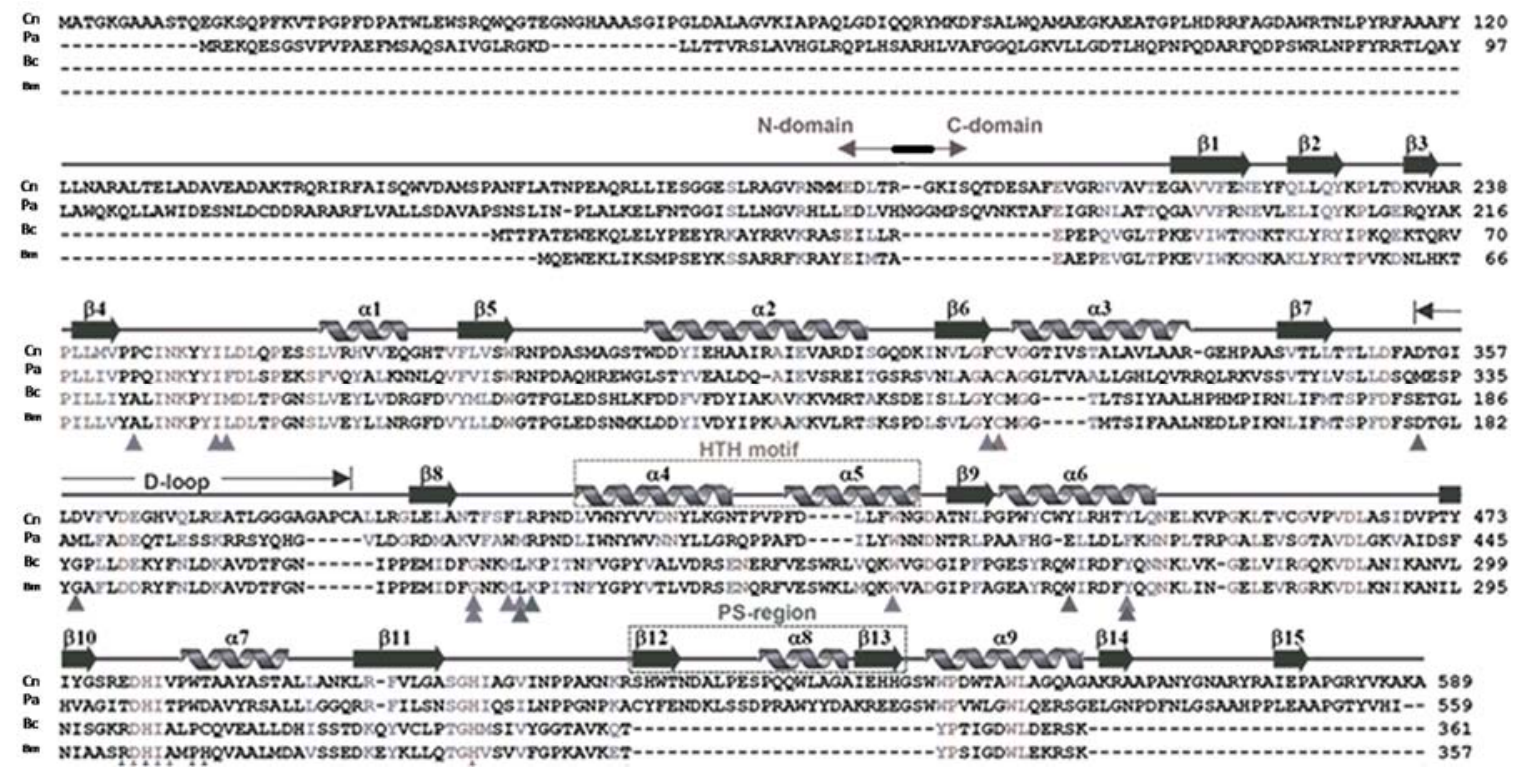

Figure 1. Amino acid sequence and secondary structure of Cupriavidus necator $\mathrm{PhaC}_{\mathrm{Cn}}$, aligned with the PHA synthase of Class II (P. aeruginosa), Class IVm and Class IVb, from Bacillus megaterium and B. cereus, respectively.

The PhaC synthase from Chromobacterium spp., PhaCPhaC ${ }_{\mathrm{Cs}}$, was extensively studied $[16,17]$. $\mathrm{PhaC}_{\mathrm{Cs}}$ has a peculiarity of utilization of $3 \mathrm{HB}, 3 \mathrm{HV}$ and $3 \mathrm{HH}$, producing scl-PHA polymers with mixed composition, with the ability to incorporate $\mathrm{C} 5$ and $\mathrm{C} 6$ alkanes into the PHA polymer. $\mathrm{PhaC}_{\mathrm{Cs}}$ was found to be highly active, with fast polymerization rate [17]. $\mathrm{PhaC}_{\mathrm{Cs}}$ is shorter in length (by about 29 amino acids) with respect to $\mathrm{PhaC}_{\mathrm{Cn}}$ and this produces differences in the numbering of amino acids. The $\mathrm{PhaC}_{\mathrm{Cs}}$ structure has a substrate-binding site hidden by a partially disordered protein domain, the CAP domain $[16,17]$. Cysteine 291 , at the end of the $B 6$-sheet, is followed by the CAP domain, containing the LID structure, which closes the accessibility of the substrate access pocket. The $\beta$-sheets in the CAP domain have been renumbered with Greek letters in the $\mathrm{PhaC}_{\mathrm{Cs}}$ sequence. Thus, from the $\mathrm{N}$-terminal sequence, up to the $\beta 6-\alpha 3$ turn, the two PHA synthases conserve the same numbering in their secondary structures, but the successive $\alpha-\beta$ turns are differently numbered. In $\mathrm{PhaC}_{\mathrm{Cs}}$, the core subdomain contains six $\beta$-strands ( $\beta 8$ to $ß 13$ ) and four a-helices ( $\alpha 4$ to $\alpha 7$ ), whose numbers do not correspond to those in the $\mathrm{PhaC}_{\mathrm{Cn}}$-CAT structure.

In $\mathrm{PhaC}_{\mathrm{Cs}}$, the catalytic triad is composed of $\mathrm{Cys}_{291}$, $\mathrm{His}_{477}$ (located after the $\beta 9$-sheet, with respect to the $\beta 11$-sheet in $\mathrm{PhaC}_{\mathrm{Cn}}$ ) and $\mathrm{Asp}_{447}$ (located at the turn formed by the $\beta 8$-strand and $\alpha 4$-helix, the $\beta 8-\alpha 4$ fold, in respect to the $\beta 10-\alpha 7$ fold in $\left.\mathrm{PhaC}_{\mathrm{Cn}}\right)$.

In other species, such as Delftia acidovorans (previously Comamonas acidovorans), the PHA synthase contains a large insert of 40 amino acid residues shown to improve the specific activity of the enzyme, located in the $\alpha / \beta$-hydrolase fold, following the catalytic cysteine after the $\beta 6$ turn [23].

Aeromonas spp., such as A. caviae, A. hydrophila and A. punctata, possess PhaC enzymes belonging to Class I. These organisms have synthases of type I with mixed substrate range, which enable the production of "Nodex" mixed-type scl-mcl-PHAs by these bacteria [24]. 
The enzyme PhaC $\mathrm{Cc}_{\mathrm{Cc}}$ from Caulobacter crescentus (C. vibrioides) [25] was displayed to accommodate 3-hydroxyalkanoates with various alkyl side-chain lengths.

Class II PhaC synthases have been extensively studied, and are widely distributed in bacteria: in Pseudomonas spp. (P. putida, P. mendocina, P. oleovorans, P. campisalis, P. stutzeri), there are two $\mathrm{PhaCPhaC}$ genes, of which $\mathrm{PhaC} 1$ is the active enzyme under physiological conditions. PhaC synthases have been reported in Halomonas spp., such as H. campisalis, Halomonas sp. O-1 and Halomonas elongata DSM2581 [26], and in P. stutzeri, and they can be exploited in polymerization of mcl-PHAs [27]. There are two PHA synthases, $\mathrm{PhaC} 1$ and $\mathrm{PhaC} 2$, in P. oleovorans, of which PhaC2 has a higher affinity for 3-hydroxyhexanoate (3HH) monomers.

Class II PhaCPhaC enzymes differ from $\mathrm{PhaC}_{\mathrm{Cn}}$, as prototype of Class I PHA synthases, by about 28 amino acids, reaching the C-terminal (1-559) with a sequence shorter by about 30 amino acids. The catalytic triad has been renumbered as $\mathrm{Cys}_{296}, \mathrm{Asp}_{452}, \mathrm{His}_{453}$ and $\mathrm{His}_{480}$ in Pseudomonas spp., prototype for Class II PHA synthases.

\subsection{Class III and Class IV PHA Synthases}

Class III PHA synthases are made of two subunits, namely, a catalytic subunit PhaC (40-53 kDa) and a second subunit PhaE (ranging from 20 to $40 \mathrm{kDa}$ ), which form the PhaEC complex, in which the PhaE subunit is necessary for PHA polymerization. Class III PhaC are structured as tetramers, such as phaEC from Chromatium vinosum (with catalytic triad $\mathrm{Cys}_{149}, \mathrm{Asp}_{302}, \mathrm{His}_{331}$ ), whose enzyme activity has been studied using substrate analogs [15]. The authors performed molecular docking and in-silico studies that are in agreement with the crystal structure of synthases available [15], based on homology models built using CPHmodels3.0, SWISS-MODEL and I-TASSER, performing structure-guided sequence profiles. The results of their analysis, referring to $\mathrm{PhaC}_{\mathrm{Cn}}$ and PhaC Class III from Chromatium vinosum, describe the presence of an active site of cysteine that is buried in a pocket. The authors, by comparison with other enzymes with known crystal structures (lipases), postulated the presence of the substrate-entrance and product-exit channels [15].

An interesting enzyme representative of Class III has been isolated and characterized in Thiocapsa pfennigii [28]. There is also an archaeal type, PhaC Class IIIA [29]: this group is represented by Haloarcula marismortui. Archaea present good perspectives of exploitation for polymer production, given by the easiness of PHB extraction.

Class IV PHA synthases from Bacillus spp. are composed of a catalytic subunit PhaC (41.5 kDa) and a PhaR (22 kDa) subunit, similarly to Class III synthases composed of PhaE and PhaC units. Class IV PHA synthases are classified as Bacillus cereus type (IV $\mathrm{C}_{\mathrm{c}}$ [30], B. megaterium type (IVm) [31], and B. bataviensis type $\left(\mathrm{IV}_{\mathrm{b}}\right)$, with $33 \%$ homology to the other PhaC sequences [5]. In E. coli expressing PhaRC from B. cereus YB-4, the biosynthesized PHA undergoes synthase-catalyzed alcoholytic cleavage using endogenous and exogenous alcohols. This alcoholysis is thought to be shared among Class IV synthases, and this reaction is useful for regulation of PHA molecular weight and for modification of the PHA carboxy terminus.

The catalytic cysteine in the active site is $C_{151}$ in B. cereus, and $C_{147}$ in B. megaterium type IV enzymes. As shown for PhaCYB4 from B. cereus YB-4, the involvement of $\mathrm{Cys}_{151}, \mathrm{Asp}_{306}$ and $\mathrm{His}_{335}$ in polymerization activity was shown by site-directed mutagenesis [5].

\subsection{Diversity and Spread of PhaC in Bacteria}

PHA synthase genes can be identified in environmental bacterial strains for a preliminary screening, before knowledge of PHA synthesis ability due to the presence of the gene, using PCR amplification with conserved primers [7,32]. Through PCR analyses, PhaC genes were detected in a collection of bacterial strains isolated from soils and from marine environments. In samples of environmental strains, we amplified $P h a C$ gene sequences in colonies from environmental isolates, and performed DNA sequencing of ribosomal DNA to identify the strains at species level: with this method, several species were classified for their ability to produce PHA, for instance, P. oleovorans, 
P. fluorescens, P. sihuiensis, P. putida, Comamonas testosteroni, Aeromonas hydrophila, as well as Cupriavidus necator. It is envisaged that PCR screening using various primer sets can be optimized to find new PhaC polymorphisms and potential novel PHA synthase sequences. Quelas reported the presence in Bradyrhizobium japonicum USDA110 of five polyhydroxyalkanoate (PHA) synthases (PhaC), distributed into four different PhaC classes [33], and characterized the requirements for two of the genes in legume nodules under various physiological conditions.

\section{Crystal Structure}

In two publications that appeared almost simultaneously, two teams reported on the crystal structure of $\mathrm{PhaC}_{\mathrm{Cn}}$-CAT, the catalytic domain of PhaC from Cupriavidus necator $[18,19,24]$. $\mathrm{PhaC}_{\mathrm{Cn}}$-CAT was shown to dimerize, and to adopt a partially open form maintaining a narrow substrate access to the active site. PhaC $\mathrm{Cn}_{\mathrm{n}}$ needs PhaM, the primer of PHA synthesis, to start and accelerate polymer synthesis, and this may be due to increased accessibility of 3HB-CoA substrate to the active site. Wittenborn obtained the crystal structure of $\mathrm{PhaC}_{\mathrm{Cn}}\left(\mathrm{C}_{319} \mathrm{~A}\right)$, a construct in which the active site cysteine $\left(\mathrm{Cys}_{319}\right)$ was mutated to alanine to improve protein stability in the absence of detergent. During the crystallization, proteolysis of $\mathrm{PhaC}_{\mathrm{Cn}}$ occurred after the $\mathrm{N}$-domain $\left(\mathrm{R}_{192}\right)$, leading to the crystal structure of the C-domain: PhaC $\mathrm{Cn}_{n}$-CAT is formed by two core subdomains $\left(\mathrm{G}_{143}-\mathrm{F}_{352}\right.$, $\left.\mathrm{L}_{450}-\mathrm{A}_{589}\right)$, flanking on both sides a dimerization domain $\left(\mathrm{A}_{353}-\mathrm{L}_{549}\right)$ containing the dimerization loop (D-loop) and the helix-loop-helix (HTH) domain; in addition, in the terminal core subdomain there is an extended C-terminal region (EC: $\mathrm{R}_{521}-\mathrm{A}_{589}$ ), which is missing in Class IV PhaC (Figure 1), and a protruding structure, $\mathrm{PS}$, that elongates from the extended C-region.

The catalytic domain of $\mathrm{PhaC}_{\mathrm{Cn}}$ contains the residues 201-368 and 378-589 (with residues 369-377 devoid of any structure), showing an $\alpha / \beta$-hydrolase fold, featuring a central mixed $\beta$-sheet flanked by $\alpha$-helices on both sides. This architecture is similar to that seen in lipases. The CAT domain in the $\mathrm{PhaC}_{\mathrm{Cn}}$ sequence is structured by the presence of the $\beta 1-4$-sheets, the $\alpha 1$-helix, the $\beta 5$-sheet, $\alpha 2$-helix and $\beta 6$-sheet facing the lipase box, followed by the $\alpha 3-\beta 7$ fold: after this structure there is the D-loop; after the $\beta 8$-sheet, there is the helix-loop-helix (HTH), composed of the $\alpha 4$ - and $\alpha 5$-helices facing each other, and the $\beta 9-\alpha 6$ fold, where the dimerization subdomain ends $\left(\mathrm{L}_{449}\right)$; as for the other amino acids of the catalytic triad, the aspartate is located between the $\beta 10-\alpha 7$ fold, and the histidine is located after the $\beta 11$-sheet. The active site of $\mathrm{PhaC}_{\mathrm{Cn}}$ is accessible via a water-filled channel, with a size of 12.5 Angstroms, which can accommodate the 3HB-CoA substrate and/or short PHA oligomers.

Two PhaC monomers interact through the dimerization surfaces $\left(\mathrm{A}_{353}-\mathrm{E}_{445}\right)$, containing hydrophobic amino acids, by means of interaction between one monomer helix-loop-helix motif (HTH) and the D-loop of the second monomer $[18,19]$.

In the report on the crystal structure obtained from the catalytic domain of PhaC from Chromobacterium sp. USM2, $\mathrm{PhaC}_{\mathrm{Cs}}$-CAT was compared to the PhaC $\mathrm{Cn}_{\mathrm{C}}$-CAT crystal structure [17]. Considering the two structures described, in $\mathrm{PhaC}_{\mathrm{CS}}-\mathrm{CAT}$, a difference in the accessibility of the active site has been evidenced. Chek showed that in the PhaC $\mathrm{Cs}_{\mathrm{Cs}}-\mathrm{CAT}$ dimer, the CAP and LID domains close the access to the substrate binding site [17]. The structure proposed by Chek and colleagues describing a PhaC $\mathrm{Cs}_{\mathrm{Cs}}$ active site covered by the CAP subdomain differs from the partially open form of the PhaC $\mathrm{C}_{\mathrm{Cn}}$ catalytic domain reported by Wittenborn. The CAP domain occupies partially the access to the substrate binding pocket, and the LID domain needs to slide away in order to free the access for $3 \mathrm{OH}$-alkanoyl-S-CoA units. Both catalytic domains of $\mathrm{PhaC}_{\mathrm{Cs}}$ and $\mathrm{PhaC}_{\mathrm{Cn}}$ form a dimer mediated by the CAP subdomain. The difference between the closed and partially open forms is provided by the conformation of the CAP subdomain. The CAP subdomain undergoes a conformational change during catalytic activity with rearrangement of the dimeric form.

The main difference between the two crystal structures was found in the folding of $\alpha \mathrm{B}^{\prime}$ - and $\eta B^{\prime}$-helices and their linker loop of $\mathrm{PhaC}_{\mathrm{Cs}}-\mathrm{CAT}$, while the corresponding positions in $\mathrm{PhaC}_{\mathrm{Cn}}-\mathrm{CAT}$ show a long $\alpha 4$-helix that presents a partial access to the active site. The region $\mathrm{Leu}_{402}-\mathrm{Asn}_{415}$ 
forming the $\alpha 4$-helix in PhaC $\mathrm{Cn}$-CAT is conserved among Class I and II PHA synthases, whereas the corresponding segment, $\mathrm{Leu}_{369}-\mathrm{Lys}_{382}$ of $\mathrm{PhaC}_{\mathrm{Cs}}-\mathrm{CAT}$, displays a disordered structure.

\section{Catalytic Mechanism}

The models proposed for the available PhaC structures hypothesize the presence of a substrate entrance tunnel that accommodates HB-CoA, with a size of about $12.5-13 \AA$, and a product egress tunnel, positioned perpendicularly to the entrance tunnel. Various catalytic mechanisms for PHA synthases have been proposed, in the context of dimerization of PHA synthases of Class I and II [17].

One mechanism is referred to as the nonprocessive ping-pong model: this mechanism requires two cysteines in the active sites in the dimer, for PHA chain elongation, with chain transfer from one cysteine to the second active site. The ping-pong mechanism requires two thiol groups located at a distance short enough to shuttle back and forth the growing ( $3 \mathrm{HB}) n$ chain between the two thiols. The dimeric structures described by Wittenborn and by Kim for PhaC $\mathrm{Cn}_{\mathrm{C}}-\mathrm{CAT}$, and by Chek for $\mathrm{PhaC}_{\mathrm{Cs}}$, show that the two active sites are too distant (33 and 28.1 Angstroms, respectively) for successive chemical reactions.

The distance between the active sites in the dimer seems to favor the mechanism based on a single active site for each elongation reaction. In the model described by Chek, the dimer, composed by two units of $\mathrm{PhaC}_{C S}$ through the contacts between the two CAP domains and the two N-domains, presents two channels leading to the two active sites. The dimeric structure proposed by Chek [17] favors the involvement of one active site for each processing step. In the model, the substrate enters the substrate-binding tunnel, while the chain product is elongated along a path near the protein surface, with a sliding mechanism of the PHA polymer under synthesis along a V-shaped cavity within the enzyme. In the proposed structure, the enzyme moves along the extremity of the forming polymer to add new $3 \mathrm{HB}$ units, rather than hosting the polymer into a product egress channel. The mechanism involves a processive model that requires a single active site for PHA chain elongation and a noncovalent intermediate, in addition to a covalent intermediate bound to the Cys residue at the active center during the catalytic cycle. The enzyme dimer, through interactions with other partners, substrates, phasins and phaM, moves the CAP domains to flip away, opening the active site entrance, and freeing the product channel, and the two core units simultaneously accept the substrate and produce the $3 \mathrm{HBn}$ polymers. The process occurs with a two-step catalysis mechanism that allows the intermediates to be located in the enlarged cavities partially freed from the CAP occupancy. The arrangement of the dimer, different from that of the PhaCCn-CAT dimer, may allow the CAP subdomains to undergo a conformational change during catalytic activity with rearrangements in the dimer that facilitates substrate entry, intermediate product formation, and product exit from the active site. According to the crystal structure of the $\mathrm{PhaC}_{\mathrm{Cn}}$-CAT dimer $[18,19]$, the substrates enter through the substrate-binding tunnel: the first $3 \mathrm{HB}-\mathrm{CoA}$ is attacked by the nucleophilic $\mathrm{Cys}-\mathrm{SH}$ to produce a 3HB-Cys covalent bond, as in the aforementioned model, and frees $\mathrm{CoA}-\mathrm{SH}$, which is released from the product egress tunnel. A second 3HB-CoA attacks 3HB-Cys thioester bond with the hydroxyl group in $3 \mathrm{HB}$ to produce a (3HB)2-CoA intermediate, a reaction that frees the Cys residue in the active center. The Cys residue again attacks the thioester bond of the (3HB)2-CoA intermediate to produce $(3 \mathrm{HB})_{n+1}$, covalently bound to the Cys residue, and releases free CoA. In this model, the growing $3 \mathrm{HB}$ polymer is bound to the enzyme at the end of each cycle. This model cannot allow it to position large molecules such as the (3HB)n-CoA intermediate within the substrate binding site, which has a cavity of 12.5 Angstroms.

An alternative model has been proposed with a succession of slightly different reactions. The model proposed for $\mathrm{PhaC}_{\mathrm{Cn}}$, by Wittenborn, implies that newly entered 3HB-CoA produces $3 \mathrm{HB}-\mathrm{Cys}$; then, $(3 \mathrm{HB})_{2}-\mathrm{CoA}$ enters the active site to produce $(3 \mathrm{HB})_{3}-\mathrm{CoA}$, which is again released from the active site. When a new $(3 \mathrm{HB})_{2}$-CoA substrate binds, the 3HB 3-hydroxyl group is deprotonated by $\mathrm{His}_{508}$, facilitated through modulation of the histidine basicity by Asp 480 . The newly formed HB alkoxide attacks the Cys-HB thioester, generating a noncovalent, CoA-bound intermediate. However, 
if the $(3 \mathrm{HB})_{3}-\mathrm{CoA}$ produced is held in the active site and attacked by the active Cys residue again to produce $(3 \mathrm{HB})_{3}$-Cys, chain elongation would then require an intersubunit reaction. Again, $(3 \mathrm{HB})_{n}-\mathrm{Cys}$ adducts would require a larger active site cavity.

\section{Mutation and Amino Acid Substitution Studies}

Several studies focused on PHB synthases with mutations enabling the enzymes to accelerate the reaction kinetics [34-37] and ability to accept bulk substrates as precursors for the production of mcl-PHAs and grafted copolymers.

Nomura and Taguchi [37] reviewed the attempts to engineer various classes of PHA synthases, either by mutagenesis or by evolution, in Class I and Class II enzymes. The methods utilized either random mutagenesis, intragenic suppression mutagenesis, gene shuffling, random mutagenesis combined with site-specific saturation mutagenesis and recombination, localized semirandom mutagenesis, PCR-mediated random chimeragenesis, intragenic suppression mutagenesis, or site-specific saturation mutagenesis.

Many authors described mutations in amino acids positioned in various domains of different PHA synthases, most often finding a decrease in production of mcl-PHA and higher synthesis of scl-PHA. Beneficial effects of mutagenesis studies of $\mathrm{Glu}_{130}$ and $\mathrm{Ser}_{477}$ have been described [38-41]. For instance, the $\mathrm{E}_{130} \mathrm{D}$ substitution and $\mathrm{S}_{477} \mathrm{X}$ mutation in type II PHA synthase showed an enhancement of PHA production and alteration of polymer molecular weight.

A mutagenesis study of Class I PHA synthases showed that the $\mathrm{F}_{420} \mathrm{~S}$ mutation in $\mathrm{PhaC}_{\mathrm{Cn}}$ increased the specific activity with a shortened lag phase [42]. This residue corresponds to $\mathrm{Phe}_{387}$ of $\mathrm{PhaC}_{\mathrm{Cs}}$, which is conserved among Class I and II PHA synthases, and is located in the $\alpha \mathrm{C}$-helix of the CAP domain. Phe 387 is involved in dimerization by participating in an intermolecular nonpolar interaction linking the $\alpha \mathrm{C}$-helix to the LID region, suggesting that the mutation may affect the conformational stability and/or conformation transition of the LID region.

The CAP subdomain provides $\alpha \mathrm{C}$ - and $\alpha \mathrm{D}$-helices as building blocks of the active site cavity filled with a cluster of water molecules. In the structure obtained by Chek [17], the C-terminal portion of the LID region of the CAP subdomain is disordered and is followed by $\alpha \mathrm{C}$-helix docked to the core subdomain. Two highly conserved residues, $\operatorname{Tr}_{392}$ and $\mathrm{Asp}_{395}$, are present in the $\alpha \mathrm{C}$-helix. $\operatorname{Tr}_{392}$ of PhaCCs is located in the $\alpha \mathrm{C}$-helix of the CAP subdomain and faces site B of the channel.

A mutational study of $\mathrm{PhaC}_{C S}$ reported by Chuah [43] showed that in $\mathrm{PhaC}_{C s}, \mathrm{Ala}_{479}$ is a critical residue required for substrate specificity, as determined by various site-specific mutational assays both in vivo and in vitro, and production tests of copolymers such as $\mathrm{P}(3 \mathrm{HB}-\mathrm{co}-3 \mathrm{HHx})$. In $\mathrm{PhaC}_{\mathrm{Cn}}$ and in other Class I enzymes, this position corresponds to the conserved residue Ala517. In the structure proposed by Chek, Ala 479 is located within the $\alpha 5$-helix, and the side chain protrudes into a depression of the molecular surface formed by loops ( $\beta 4-\alpha 1, \beta 9-\alpha 5$ and $\alpha 5-\beta 10$ loops) from the core subdomain, and is partially covered by the helix $\eta \mathrm{B}^{\prime}$ and the following loop of the LID region from the CAP subdomain. The $\mathrm{A}_{479}$ mutation results in weakening of the interactions between the LID region and the core subdomain, and stabilizes the active form of this enzyme by releasing the LID region from the active site. Since $\mathrm{Ala}_{479}$ is surrounded by polar residues $\left(\mathrm{Ser}_{475}\right.$ and $\operatorname{Arg}_{490}$ ), it is supposed that replacement of $\mathrm{Ala}_{479}$ with Ser or Thr facilitates hydrogen-bonding interactions with the polar residues and stabilization of the $\alpha 5$-helix harboring the active residue His 477 , important for enzyme activity.

Amara and Rhem attempted to modify the activity of PhaC from Pseudomonas species [40]. The conserved residue $\operatorname{Trp}_{398}$ was replaced, for example, to $\operatorname{Tr}_{398}$ Phe and $\operatorname{Trp}_{398} \mathrm{Ala}$, and the mutation resulted in inactivation of the enzyme. Using the threading model of enzyme structure, the authors located the Trp residue as exposed on the surface, in agreement with the results shown by Chek for Class I enzymes [44-49].

$\mathrm{Tyr}_{412}$ in $\mathrm{PhaC}_{\mathrm{Cs}}$, and $\mathrm{Tyr}_{446}$ in the $\alpha 6$-helix in $\mathrm{PhaC}_{\mathrm{Cn}}$, are residues conserved in Class I, III and IV PHA synthases, while Phe occupies this position in Class II synthases; in addition to this amino acid position, there is a second substitution that seems to have a role in accommodating larger substrates. 
$\mathrm{Tyr}_{438}$ is conserved in Class I, III and IV enzymes, while in Class II PhaC this position is occupied by His: this may contribute to a reduction of size, eliminating the bulky side chain (phenol ring), and determining changes in polar interactions with other amino acids facing the substrate entrance tunnel; these amino acids' interactions may account for the property to accommodate large substrates in Class II enzymes.

PhaC1 and PhaC2 from Pseudomonas stutzeri [42] have been applied to produce mcl-PHAs in engineered bacteria. PsPhaC2 with four point mutations, at $\mathrm{E}_{130} \mathrm{D}, \mathrm{S}_{325} \mathrm{~T}, \mathrm{~S}_{477} \mathrm{G}$ and $\mathrm{Q}_{481} \mathrm{~K}$, was used to accommodate substrates with various shapes and structures, to produce mcl-PHAs and block copolymers. The putative catalytic residues $\mathrm{Cys}_{296}, \mathrm{Asp}_{452}, \mathrm{His}_{453}$ and His 480 were replaced by site-specific mutagenesis $[38,39]$. Considering the Pseudomonas mcl-PHA synthases, the His ${ }_{480} \mathrm{Gln}$ substitution did not affect enzyme activity, posing the doubt that His is not a component of the catalytic triad. As for a second conserved histidine, when $\mathrm{His}_{453}$ was replaced by Gln, the modified enzyme showed only $24 \%$ of wild-type in-vivo activity, which makes one suppose that $\mathrm{His}_{453}$ might be part of the catalytic triad in Class II PHA synthases [40]. However, no other study confirmed the involvement of $\mathrm{His}_{453}$ in Class II PhaC2 catalysis.

Sheu studied the increase of PHA synthase thermostability and activity, using chimeric constructs, indicating that some amino acid substitutions may stabilize the enzyme at higher temperature [34].

\section{Production of PHA in Fermentors}

Various companies are involved in production of bioplastics for industrial applications. The methods are various, either using patented strains, engineered PHA synthases, or growth conditions favoring the high yield and high PHA content/dry cell weight. Recently, a fine review has been published on this topic [50]. In the field of monitoring the endpoint step of PHA synthesis, and bacteria collection, various methods have been established, from lipid staining [51] and analysis of fluorescence intensity, to physic-chemical analyses (Raman, FTIR spectra). Since bacterial cultures require sterilization that is costly at industrial scale, methods based on halophilic strains have been proposed to circumvent the sterilization process. Extraction of PHAs from bacteria requires costly procedures, and therefore, researchers used archaea or cyanobacteria that have PHA granules easily extracted, decreasing the costs of production $[52,53]$.

\section{Progress and Advancements in the PHA Field}

Recent advancements in PHA granule structure and composition have been achieved [54].

PHA granules harbour a considerable number of proteins on their polymer surface, suggesting that they represent supramolecular complexes with specific functions. The high-molecular-weight storage PHB consists of $>10^{3} 3 \mathrm{HB}$ residues (storage PHB). PHB granules in vivo are covered by a surface layer that is distinct from the polymer core. PHA granules are structured through the action of various proteins on the surface. As an analogy to organelles, the functional granules were proposed to be named carbonosomes by Jendrossek and Pfeiffe, although this term has not been widely accepted. PHA granules represent supramolecular complexes with specific functions. In addition to phasins (such as PhaP2, PhaP3, PhaP4), among the proteins identified during PHA granule isolation, there are the PHB synthase (PhaC1), PhaM, the activator of PhaC, acetyl-CoA acetyltransferase, and acyl-CoA synthetase: their presence may be explained by the need to avoid accumulation of $\mathrm{CoA}-\mathrm{SH}$, produced by the PHA synthase during polymer synthesis, since an excess of CoA would inhibit the enzyme. The most accurate model for PHA synthesis within bacterial cells is the Scaffold Model: it assumes that PHB synthase of nascent PHB granules is attached to a scaffold within the cell. PHB granules have been localized in the cell centre, along with the length axis of the bacteria. PhaM, which specifically interacts with PhaC1 and with phasin PhaP5, interacts also with DNA and with the nucleoid in vitro and in vivo, and this may explain why PHB granules have been found attached to the bacterial nucleoid. 


\section{Conclusions}

In this review, we reported on the classification of PHA synthases, the proposed structures and roles of individual amino acids in the catalysis, and mechanism of activity of Class I and Class II PHA synthases, presenting the information available on the other types of enzymes. We have reviewed the engineering attempts and the effect of modification of key amino acids on the enzymatic activity and product formation. It is expected that PHA synthases may be further improved to produce effectively and at convenient costs tailor-made polymers.

Author Contributions: V.M. and O.F.D. provided the information on research data on environmental strains. P.P. wrote and reviewed the manuscript.

Funding: This research received no external funding.

Acknowledgments: No funds have been provided for covering the costs to publish in open access.

Conflicts of Interest: The authors declare no conflict of interest.

\section{References}

1. Kumar, P.; Jun, H.B.; Kim, B.S. Co-production of polyhydroxyalkanoates and carotenoids through bioconversion of glycerol by Paracoccus sp. strain LL1. Int. J. Biol. Macromol. 2018, 107, 2552-2558. [CrossRef] [PubMed]

2. Park, D.H.; Kim, B.S. Production of poly(3-hydroxybutyrate) and poly(3-hydroxybutyrateco-4-hydroxybutyrate) by Ralstonia eutropha from soybean oil. New Biotechnol. 2011, 28, 719-724. [CrossRef] [PubMed]

3. Kumar, P.; Singh, M.; Mehariya, S.; Patel, S.K.; Lee, J.K.; Kalia, V.C. Ecobiotechnological approach for exploiting the abilities of Bacillus to produce co-polymer of Polyhydroxyalkanoate. Indian J. Microbiol. 2014, 54, 151-157. [CrossRef] [PubMed]

4. Hermann-Krauss, C.; Koller, M.; Muhr, A.; Fas, H.; Stelzer, F.; Braunegg, G. Archaeal production of polyhydroxyalkanoate (PHA) co- and terpolyesters from biodiesel industry-derived by-products. Archaea 2013, 2013, 129268. [CrossRef] [PubMed]

5. Tsuge, T.; Hyakutake, M.; Mizuno, K. Class IV polyhydroxyalkanoate (PHA) synthases and PHA-producing Bacillus. Appl. Microbiol. Biotechnol. 2015, 99, 6231-6240. [CrossRef] [PubMed]

6. Ansari, S.; Fatma, T. Cyanobacterial polyhydroxybutyrate (PHB): Screening, optimization and characterization. PLoS ONE 2016, 11, e0158168. [CrossRef] [PubMed]

7. Tan, G.-Y.A.; Chen, C.-L.; Li, L.; Ge, L.; Wang, L.; Ningtyas Razaad, I.M.; Li, Y.; Zhao, L.; Mo, Y.; Wang, J.-Y. Start a research on biopolymer polyhydroxyalkanoate (PHA): A review. Polymers 2014, 6, 706-754. [CrossRef]

8. Poltronieri, P.; Mezzolla, V.; D'Urso, O.F. PHB production in biofermentors assisted through biosensor applications. Proceedings 2017, 1,4 . [CrossRef]

9. Chen, G.-Q.; Hajnal, I.; Wu, H.; Lv, L.; Ye, J. Engineering biosynthesis mechanisms for diversifying Polyhydroxyalkanoates. Trends Biotechnol. 2015, 33, 565-574. [CrossRef] [PubMed]

10. Chen, G.-Q.; Hajnal, I. The 'PHAome'. Trends Biotechnol. 2015, 33, 559-564. [CrossRef] [PubMed]

11. Le Meur, S.; Zinn, M.; Egli, T.; Thöny-Meyer, L.; Ren, Q. Production of medium-chain-length polyhydroxyalkanoates by sequential feeding of xylose and octanoic acid in engineered Pseudomonas putida KT2440. BMC Biotechnol. 2012, 12, 53. [CrossRef] [PubMed]

12. Anjum, A.; Zuber, M.; Zia, K.M.; Noreen, A.; Anjum, M.N.; Tabasum, S. Microbial production of polyhydroxyalkanoates (PHAs) and its copolymers: A review of recent advancements. Int. J. Biol. Macromol. 2016, 89, 161-174. [CrossRef] [PubMed]

13. Meng, D.C.; Shen, R.; Yao, H.; Chen, J.C.; Wu, Q.; Chen, G.Q. Engineering the diversity of polyesters. Curr. Opin. Biotechnol. 2014, 29, 24-33. [CrossRef] [PubMed]

14. Mezzolla, V.; D'Urso, O.F.; Poltronieri, P. Optimization of polyhydroxyalkanoate production by recombinant E. coli supplemented with different plant by-products. Biotechnol. Indian J. 2017, 13, 138.

15. Zhang, W.; Chen, C.; Cao, R.; Maurmann, L.; Li, P. Inhibitors of polyhydroxyalkanoate (PHA) synthases: Synthesis, molecular docking, and implications. ChemBioChem 2015, 16, 156-166. [CrossRef] [PubMed] 
16. Bhubalan, K.; Chuah, J.A.; Shozui, F.; Brigham, C.J.; Taguchi, S.; Sinskey, A.J.; Rha, C.; Sudesh, K. Characterization of the highly active polyhydroxyalkanoate synthase of Chromobacterium sp. strain USM2. Appl. Environ. Microbiol. 2011, 77, 2926-2933. [CrossRef] [PubMed]

17. Chek, M.F.; Kim, S.Y.; Mori, T.; Arsad, H.; Samian, M.R.; Sudesh, K.; Hakoshima, T. Structure of polyhydroxyalkanoate (PHA) synthase PhaC from Chromobacterium sp. USM2, producing biodegradable plastics. Sci. Rep. 2017, 7, 5312. [CrossRef] [PubMed]

18. Wittenborn, E.C.; Jost, M.; Wei, Y.; Stubbe, J.; Drennan, C.L. Structure of the Catalytic Domain of the Class I Polyhydroxybutyrate Synthase from Cupriavidus necator. J. Biol. Chem. 2016, 291, 25264-25277. [CrossRef] [PubMed]

19. Kim, J.; Kim, Y.-J.; Choi, S.Y.; Lee, S.Y.; Kim, K.-J. Crystal structure of Ralstonia eutropha polyhydroxyalkanoate synthase C-terminal domain and reaction mechanisms. Biotechnol. J. 2017, 12, 1600648. [CrossRef] [PubMed]

20. Kumar, P.; Ray, S.; Kalia, V.C. Production of co-polymers of polyhydroxyalkanoates by regulating the hydrolysis of biowastes. Bioresour. Technol. 2016, 200, 413-419. [CrossRef] [PubMed]

21. Mifune, J.; Nakamura, S.; Fukui, T. Targeted engineering of Cupriavidus necator chromosome for biosynthesis of poly(3-hydroxybutyrate-co-3-hydroxyhexanoate) from vegetable oil. Can. J. Chem. 2008, 86, 621-627. [CrossRef]

22. Kim, Y.-J.; Choi, S.Y.; Kim, J.; Jin, K.S.; Lee, S.Y.; Kim, K.-J. Structure and function of the N-terminal domain of Ralstonia eutropha polyhydroxyalkanoate synthase, and the proposed structure and mechanisms of the whole enzyme. Biotechnol. J. 2017, 12, 1600649. [CrossRef] [PubMed]

23. Tsuge, T.; Imazu, S.; Takase, K.; Taguchi, S.; Doi, Y. An extra large insertion in the polyhydroxyalkanoate synthase from Delftia acidovorans DS-17: Its deletion effects and relation to cellular proteolysis. FEMS Microbiol. Lett. 2004, 231, 77-83. [CrossRef]

24. Lee, S.H.; Oh, D.H.; Ahn, W.S.; Lee, Y.; Choi, J.; Lee, S.Y. Production of poly(3-hydroxybutyrate-co-3-hydroxyhexanoate) by high-cell-density cultivation of Aeromonas hydrophila. Biotechnol. Bioeng. 2000, 67, 240-244. [CrossRef]

25. Qi, Q.; Rehm, B.H. Polyhydroxybutyrate biosynthesis in Caulobacter crescentus: Molecular characterization of the polyhydroxybutyrate synthase. Microbiology 2001, 147, 3353-3358. [CrossRef] [PubMed]

26. Ilham, M.; Nakanomori, S.; Kihara, T.; Hokamura, A.; Matsusaki, H.; Tsuge, T.; Mizuno, K. Characterization of polyhydroxyalkanoate synthases from Halomonas sp. O-1 and Halomonas elongata DSM2581: Site-directed mutagenesis and recombinant expression. Polym. Degrad. Stab. 2014, 109, 416-423. [CrossRef]

27. Chen, J.-Y.; Song, G.; Chen, G.-Q. A lower specificity PhaC2 synthase from Pseudomonas stutzeri catalyses the production of copolyesters consisting of short-chain-length and medium-chain-length 3-hydroxyalkanoates. Antonie Leeuwenhoek 2006, 89, 157-167. [CrossRef] [PubMed]

28. Liebergesell, M.; Rahalkar, S.; Steinbüchel, A. Analysis of the Thiocapsa pfennigii polyhydroxyalkanoate synthase: Subcloning, molecular characterization and generation of hybrid synthases with the corresponding Chromatium vinosum enzyme. Appl. Microbiol. Biotechnol. 2000, 54, 186-194. [CrossRef] [PubMed]

29. Han, J.; Hou, J.; Liu, H.; Cai, S.; Feng, B.; Zhou, J.; Xiang, H. Wide distribution among Halophilic Archaea of a novel Polyhydroxyalkanoate Synthase subtype with homology to bacterial type III Synthases. Appl. Environ. Microbiol. 2010, 76, 7811-7819. [CrossRef] [PubMed]

30. Kihara, T.; Hiroe, A.; Ishii-Hyakutake, M.; Mizuno, K.; Tsuge, T. Bacillus cereus-type polyhydroxyalkanoate biosynthetic gene cluster contains R-specific enoyl-CoA hydratase gene. Biosci. Biotechnol. Biochem. 2017, 81, 1627-1635. [CrossRef] [PubMed]

31. Hyakutake, M.; Tomizawa, S.; Mizuno, K.; Abe, H.; Tsuge, T. Alcoholytic cleavage of polyhydroxyalkanoate chains by class IV synthases induced by endogenous and exogenous ethanol. Appl. Environ. Microbiol. 2014, 80, 1421-1429. [CrossRef] [PubMed]

32. Montenegro, E.M.D.S.; Delabary, G.S.; Silva, M.A.C.D.; Andreote, F.D.; Lima, A.O.S. Molecular diagnostic for prospecting polyhydroxyalkanoate-producing bacteria. Bioengineering 2017, 4, 52. [CrossRef] [PubMed]

33. Quelas, J.I.; Mongiardini, E.J.; Pérez-Giménez, J.; Parisi, G.; Lodeiro, A.R. Analysis of two polyhydroxyalkanoate synthases in Bradyrhizobium japonicum USDA 110. J. Bacteriol. 2013, 195, 3145-3155. [CrossRef] [PubMed]

34. Sheu, D.S.; Chen, W.M.; Lai, Y.W.; Chang, R.C. Mutations derived from the thermophilic polyhydroxyalkanoate synthase PhaC enhance the thermostability and activity of PhaC from Cupriavidus necator H16. J. Bacteriol. 2012, 194, 2620-2629. [CrossRef] [PubMed] 
35. Takase, K.; Matsumoto, K.; Taguchi, S.; Doi, Y. Alteration of substrate chain-length specificity of type II synthase for polyhydroxyalkanoate biosynthesis by in vitro evolution: In vivo and in vitro enzyme assays. Biomacromolecules 2004, 5, 480-485. [CrossRef] [PubMed]

36. Chen, C.; Cao, R.; Shrestha, R.; Ward, C.; Katz, B.B.; Fischer, C.J.; Tomich, J.M.; Li, P. Trapping of intermediates with substrate analog HBOCoA in the polymerizations catalyzed by class III polyhydroxybutyrate (PHB) synthase from Allochromatium vinosum. ACS Chem. Biol. 2015, 10, 1330-1339. [CrossRef] [PubMed]

37. Nomura, C.T.; Taguchi, S. PHA synthase engineering toward superbiocatalysts for custom-made biopolymers. Appl. Microbiol. Biotechnol. 2007, 73, 969-979. [CrossRef] [PubMed]

38. Matsumoto, K.; Takase, K.; Aoki, E.; Doi, Y.; Taguchi, S. Synergistic effects of Glu130Asp substitution in the type II polyhydroxyalkanoate (PHA) synthase: Enhancement of PHA production and alteration of polymer molecular weight. Biomacromolecules 2005, 6, 99-104. [CrossRef] [PubMed]

39. Matsumoto, K.; Aoki, E.; Takase, K.; Doi, Y.; Taguchi, S. In vivo and in vitro characterization of Ser477X mutations in polyhydroxyalkanoate (PHA) synthase 1 from Pseudomonas sp. 61-3: Effects of beneficial mutations on enzymatic activity, substrate specificity, and molecular weight of PHA. Biomacromolecules 2006, 7, 2436-2442. [CrossRef] [PubMed]

40. Amara, A.A.; Rehm, B.H. Replacement of the catalytic nucleophile cysteine-296 by serine in class II polyhydroxyalkanoate synthase from Pseudomonas aeruginosa-mediated synthesis of a new polyester: Identification of catalytic residues. Biochem. J. 2003, 374, 413-421. [CrossRef] [PubMed]

41. Zou, H.; Shi, M.; Zhang, T.; Li, L.; Li, L.; Xian, M. Natural and engineered polyhydroxyalkanoate (PHA) synthase: Key enzyme in biopolyester production. Appl. Microbiol. Biotechnol. 2017, 101, 7417-7426. [CrossRef] [PubMed]

42. Chen, J.Y.; Liu, T.; Zheng, Z.; Chen, J.C.; Chen, G.-Q. Polyhydroxyalkanoate synthases PhaC1 and PhaC2 from Pseudomonas stutzeri 1317 had different substrate specificities. FEMS Microbiol. Lett. 2004, 234, $231-237$. [CrossRef] [PubMed]

43. Chuah, J.A.; Tomizawa, S.; Yamada, M.; Tsuge, T.; Doi, Y.; Sudesh, K.; Numata, K. Characterization of site-specific mutations in a short-chain-length/medium-chain-length polyhydroxyalkanoate synthase: In vivo and in vitro studies of enzymatic activity and substrate specificity. Appl. Environ. Microbiol. 2013, 79, 3813-3821. [CrossRef] [PubMed]

44. Normi, Y.M.; Hiraishi, T.; Taguchi, S.; Sudesh, K.; Najimudin, N.; Doi, Y. Site-directed saturation mutagenesis at residue F420 and recombination with another beneficial mutation of Ralstonia eutropha polyhydroxyalkanoate synthase. Biotechnol. Lett. 2005, 27, 705-712. [CrossRef] [PubMed]

45. Tsuge, T.; Saito, Y.; Kikkawa, Y.; Hiraishi, T.; Doi, Y. Biosynthesis and compositional regulation of poly(3-hydroxybutyrate)-co-(3-hydroxyhexanoate) in recombinant Ralstonia eutropha expressing mutated polyhydroxyalkanoate synthase genes. Macromol. Biosci. 2004, 4, 238-242. [CrossRef] [PubMed]

46. Shozui, F.; Matsumoto, K.; Sasaki, T.; Taguchi, S. Engineering of polyhydroxyalkanoate synthase by Ser477X/Gln481X saturation mutagenesis for efficient production of 3-hydroxybutyrate-based copolyesters. Appl. Microbiol. Biotechnol. 2009, 84, 1117-1124. [CrossRef] [PubMed]

47. Tsuge, T.; Watanabe, S.; Shimada, D.; Abe, H.; Doi, Y.; Taguchi, S. Combination of N149S and D171G mutations in Aeromonas caviae polyhydroxyalkanoate synthase and impacton polyhydroxyalkanoate biosynthesis. FEMS Microbiol. Lett. 2007, 277, 217-222. [CrossRef] [PubMed]

48. Gao, X.; Yuan, X.X.; Shi, Z.Y.; Guo, Y.Y.; Shen, X.W.; Chen, J.C.; Wu, Q.; Chen, G.-Q. Production of copolyesters of 3-hydroxybutyrate and medium-chain-length 3-hydroxyalkanoates by E. coli containing an optimized PHA synthase gene. Microb. Cell Fact. 2012, 11, 130. [CrossRef] [PubMed]

49. Shen, X.W.; Shi, Z.Y.; Song, G.; Li, Z.J.; Chen, G.-Q. Engineering of polyhydroxyalkanoate (PHA) synthase PhaC2Ps of Pseudomonas stutzeri via site-specific mutation for efficient production of PHA copolymers. Appl. Microbiol. Biotechnol. 2011, 91, 655-665. [CrossRef] [PubMed]

50. Koller, M. A review on established and emerging fermentation schemes for microbial production of Polyhydroxyalkanoate (PHA) biopolyesters. Fermentation 2018, 4, 30. [CrossRef]

51. Choi, J.E.; Na, H.Y.; Yang, T.H.; Rhee, S.K.; Song, J.K. A lipophilic fluorescent LipidGreen1-based quantification method for high-throughput screening analysis of intracellular poly-3-hydroxybutyrate. AMB Express 2015, 5, 131. [CrossRef] [PubMed]

52. Madkour, M.H.; Heinrich, D.; Alghamdi, M.A.; Shabbaj, I.I.; Steinbüchel, A. PHA recovery from biomass. Biomacromolecules 2013, 14, 2963-2972. [CrossRef] [PubMed] 
53. Koller, M.; Niebelschütz, H.; Braunegg, G. Strategies for recovery and purification of poly [(R)-3-hydroxyalkanoates](PHA) biopolyesters from surrounding biomass. Eng. Life Sci. 2013, 13, 549-562. [CrossRef]

54. Jendrossek, D.; Pfeiffer, D. New insights in the formation of polyhydroxyalkanoate granules (carbonosomes) and novel functions of poly(3-hydroxybutyrate). Environ. Microbiol. 2014, 16, 2357-2373. [CrossRef] [PubMed] 